\title{
Total versus Superficial Parotidectomy for Stage III Melanoma
}

Aileen P. Wertz MD ${ }^{1}$, Alison B. Durham MD², Kelly M. Malloy MD', Timothy M. Johnson $\mathrm{MD}^{2}$, Carol R. Bradford $\mathrm{MD}^{1}$, Scott A. McLean MD PhD ${ }^{1}$

Authors' Affiliations: ${ }^{1}$ Department of Otolaryngology - Head and Neck Surgery, University of Michigan Health System, Ann Arbor, MI ${ }^{2}$ Department of Dermatology, University of Michigan Health System, Ann Arbor, MI.

Acknowledgements: The authors would like to thank the University of Michigan CSCAR (Consulting for Statistics, Computing, and Analytics Research) for their assistance with data analysis.

This paper was presented at the 2016 AAO-HNSF Annual Meeting and Oto Expo on $09 / 18 / 2016$.

Corresponding Author: Aileen P. Wertz, MD

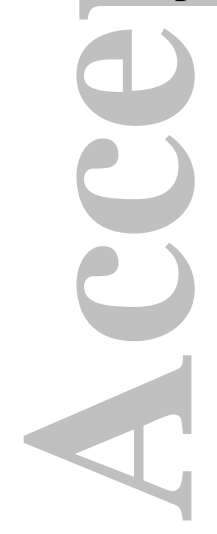

1904 Taubman Center, 1500 East Medical Center Drive Ann Arbor, MI 48109

Phone: 734-936-8051

Fax: 734-936-8052

Email: aibutera@med.umich.edu

Running title: Total versus Superficial Parotidectomy

Key words: total parotidectomy; superficial parotidectomy; malignant melanoma; facial nerve function; regional recurrence

This is the author manuscript accepted for publication and has undergone full peer review but has not been through the copyediting, typesetting, pagination and proofreading process, which may lead to differences between this version and the Version record. Please cite this article as doi:10.1002/ hed.24810. 


\section{Abstract}

Background: Our primary objective was to describe parotid recurrence rates after superficial and total parotidectomy.

Methods: A retrospective cohort study was performed on patients with cutaneous melanoma metastatic to the parotid gland who underwent parotidectomy from 1998 through 2014. Primary outcome was parotid bed recurrence. Secondary outcomes were facic facial nerve function postoperatively and at last follow-up.

Results: 129 patients were included in the study. Thirty-four (26\%) underwent total, ninety-five underwent superficial parotidectomy. Twelve (13\%) patients developed parotid bed recurrence after superficial parotidectomy alone versus zero after total $(\mathrm{p}=0.035)$. Facial nerve function, clinically detected disease, stage, and adjuvant treatment were not statistically different between groups $(p=0.32,0.32,0.13,1$, respectively).

Conclusion: Parotid bed melanoma recurrence was more common after superficial parotidectomy compared to total parotidectomy and recurrence resulted in significant facial nerve functional deficit. Our results support total parotidectomy when metastatic melanoma involves the parotid nodal basin.

John Wiley \& Sons, Inc. 


\section{Introduction}

Over 144,860 new cases of melanoma, over 76,000 of which are invasive, will be diagnosed in the United States in $2016^{1}$. Living with melanoma is common with over 1.2 million melanoma survivors estimated in the United States alone. It is the $3^{\text {rd }}$ most prevalent cancer in men and $5^{\text {th }}$ most prevalent cancer in women in the United States ${ }^{2}$. Hence, quality of life after treatment is a valid consideration. (1) While metastasis to the parotid gland is less common than to the cervical lymphatics, parotid lymphatic management is crucial as progression of parotid disease places facial nerve function at risk. The lymphatic system develops prior to the formation of the parotid capsule; therefore, lymph nodes are incorporated into the parotid parenchyma and without regard to facial nerve anatomy, as the facial nerve does not represent a fascial boundary to the lymphatic system ${ }^{3,4}$.

Cadaveric studies have demonstrated that lymph nodes are consistently present both superficial and deep to the facial nerve within the parotid gland ${ }^{5-7}$. When total parotidectomy has been performed for metastatic melanoma and other parotid malignancy, metastatic disease has been found in lymph nodes deep to the facial nerve $e^{4,8,9}$. For metastatic melanoma, a $7 \%$ parotid bed recurrence rate after superficial parotidectomy is quoted in the literature ${ }^{10}$, compared with a $0 \%$ parotid bed recurrence rate after total parotidectomy ${ }^{8}$.

Despite the evidence that melanoma does metastasize to the deep lobe of the parotid gland and that parotid bed recurrence may be reduced with total parotidectomy, superficial parotidectomy alone is the recommended treatment in the current National Comprehensive Cancer Network $(\mathrm{NCCN})$ guidelines ${ }^{11}$. While studies have reported 
recurrence rates after superficial or total parotidectomy, no study has directly compared recurrence rates after these two surgeries.

Our primary purpose was to describe parotid bed recurrence rates after superficial and total parotidectomy for stage III metastatic melanoma. The secondary aim was to describe facial nerve function immediately postoperatively and at last follow up.

\section{Materials and Methods}

Approval for this study was granted by the University of Michigan Medical School Institutional Review Board for Human Subject Research (HUM00048768). Our melanoma database was queried for patients diagnosed with primary cutaneous melanoma on the head and neck or melanoma metastatic to the parotid gland with unknown primary who underwent initial parotidectomy, either superficial or total, from 1998 through 2014. Patients were included in the study if they had biopsy-confirmed metastatic melanoma to their parotid gland either on fine-needle aspiration (FNA) (performed when a palpable mass was detected on physical examination) or sentinel lymph node biopsy (SLNB) (occult nodal disease). Primary excision, SLNB, and/or other prior excision of primary site may have been done at another institution and was not an exclusion criterion, however all parotidectomies were performed at the University of Michigan by surgeons who were members of the University of Michigan Multidisciplinary Melanoma Tumor board and regularly treated cutaneous malignancy, and/or otolaryngologists with head and neck surgery fellowship training. Total parotidectomy was defined as removal of parotid tissue deep to the facial nerve in addition to removing gland superficial to the facial nerve. Typically, superficial parotidectomy, tracing each branch of the facial nerve, was performed first. Once 
superficial parotidectomy was complete, parotid tissue deep to the facial nerve was removed by carefully dissecting glandular tissue free of the overlying facial nerve. The procedure was altered as needed if there was clinically detected disease in both the superficial and deep lobe, preventing superficial and deep parotid tissue from being removed separately while removing the tumor en-bloc.

The decision to perform total parotidectomy was surgeon dependent. One of our surgeons, Dr. McLean, underwent a practice shift and began performing total parotidectomy on all patients with melanoma metastatic to the parotid gland in 2013 . The remainder of surgeons performed total parotidectomy when there was concern for metastatic disease deep to the facial nerve. For these surgeons, the decision to perform total parotidectomy was made preoperatively or intraoperatively depending on if there was preoperative imaging or intraoperative findings suggestive of possible disease deep to the facial nerve.

Demographics and clinical course/outcome measures were confirmed via the electronic medical record by manual abstraction. Interventions compared were superficial and total parotidectomy, which was determined by review of the surgeon's operative report. Primary outcome was parotid bed recurrence, confirmed by biopsy in all cases except one confirmed with imaging. Variables were clinically detected versus occult nodal disease, stage, adjuvant treatment and length of follow up. All patients were pathologically staged using the American Joint Committee on Cancer (AJCC) cancer $7^{\text {th }}$ edition staging criteria ${ }^{12}$. Chemotherapy, immunotherapy, and radiation were all considered adjuvant treatment. Adjuvant treatment had to occur after surgery and prior to any recurrence to be included in this study. 
Secondary outcomes were facial nerve function postoperatively and at last followup. Facial nerve function was classified as House-Brackmann 1-3 or 4-6 ${ }^{13}$. Inpatient and outpatient postoperative notes were reviewed to identify postoperative facial nerve function and postoperative complications. Temporary facial asymmetry with full recovery of function was categorized based on worst deficit. If facial nerve exam was not documented postoperatively then the patient was excluded from analyses of facial nerve function. Only complications due to parotidectomy or general anesthesia were included. Complications due to neck dissection, such as cranial nerve XI injury, were not included when calculating complication rates. Complications occurring during admission and after discharge within the first 3 months postoperatively were included.

Statistical analyses were performed using excel and SPSS version 23 (ㄷ IBM, New York, USA). Fisher exact tests (for categorical variables) and Mann-Whitney U tests (for continuous variables) were used to assess statistically significant differences between patients undergoing superficial and total parotidectomy. Log-rank survival analysis was performed for parotid recurrence. A p-value of 0.05 was considered statistically significant.

\section{Results}

One hundred twenty-nine patients underwent parotidectomy for metastatic melanoma. Thirty-four (26\%) underwent total, 95 (74\%) underwent superficial parotidectomy. Median follow up was 22 months. See Table 1 for additional demographic data. Twelve (13\%) patients developed parotid bed recurrence after superficial parotidectomy versus zero after total parotidectomy (Fisher exact test $\mathrm{p}=$ 0.035; Table 1). Survival analysis, censoring patients lost to follow up, showed no 
statistically significant difference between interventions $(p=0.10$; Figure 1$)$.

Of the patients that recurred in the parotid bed, $3(25 \%)$ had clinically detected (palpable) disease prior to superficial parotidectomy. 33\% had stage IIIa disease, $17 \%$ stage IIIb, and 50\% stage IIIc. $25 \%$ had adjuvant treatment prior to parotid bed recurrence including radiation (1), interferon (1), and combination immune and chemotherapy (1). Primary tumor characteristics, nodal disease burden, and adjuvant treatment were compared between patient who did and did not recur after superficial parotidectomy (Table 2). There were no significant differences in primary tumor characteristics or adjuvant treatment. The only significant difference was in rate of N3 nodal disease $(\mathrm{p}=0.03$; Table 2$)$.

Facial nerve function, complication rate, clinically detected versus occult nodal disease, stage, and if adjuvant treatment was administered were not statistically different between groups (Table 1). When the type of adjuvant treatment was examined, significantly more patients who underwent superficial parotidectomy underwent adjuvant immune therapy with interferon (Table 1). The rate of radiation therapy, chemotherapy, and combined modality therapy was not significantly different between groups (Table 1). Interferon was the only immune therapy patients in this study received.

The average length of follow up in the superficial parotidectomy group was shorter than the total parotidectomy group ( 24 months vs. 16 months, $\mathrm{p}=0.05$; Table 1 ), which is at least partially due to more total parotidectomies performed in 2013 and 2014.

Twenty-five patients who underwent superficial parotidectomy had no postoperative facial nerve function documented and were therefore excluded from facial nerve function analyses. Two patients were House-Brackmann (HB) 4 or greater in the 
early postoperative period after superficial parotidectomy. Neither was due to intraoperatively-documented tumor on or surrounding the facial nerve. One regained complete eye closure over the first 6 months of follow up. The other had no documented improvement in facial nerve function throughout their follow up. Four patients were HB 4 or greater in the early postoperative period after total parotidectomy. All four were due to tumor encasement of the facial nerve with intraoperative sacrifice of the upper division (2) or main trunk (2). One of these patients was excluded from facial nerve function analyses because they presented preoperatively, prior to initial parotidectomy, with facial paralysis. See Table 1 for complete postoperative facial nerve function results. Of the 12 patients who had parotid bed recurrence, 10 had facial nerve function documented after recurrence, 5 of which developed complete facial nerve paralysis due to recurrent melanoma.

Complications in the total parotidectomy group were: delayed wound healing (1), rhabdomyolysis without sequelae (1), and fall with radius fracture (1). Complications in the superficial parotidectomy group were: seroma or hematoma (3), delayed wound healing (2), urinary tract infection (2), pneumonia (1), rhabdomyolysis requiring temporary hemodialysis (1), and pulmonary edema (1; Table 1).

\section{Discussion}

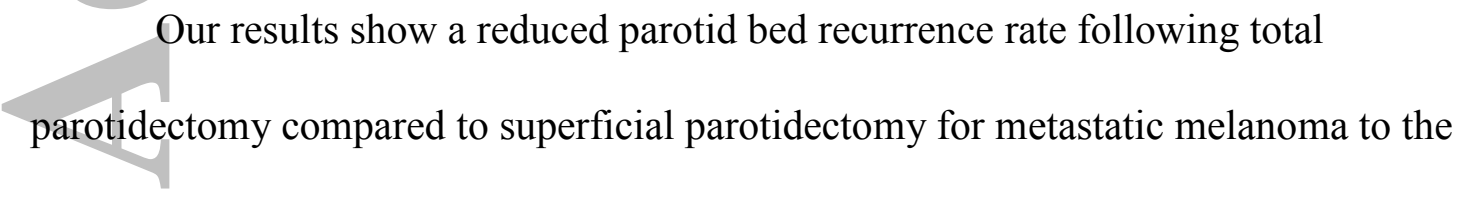
parotid gland. Additionally, no increased morbidity was associated with total parotidectomy compared to superficial parotidectomy in our cohort. These findings are consistent with the single prior report of total parotidectomy for metastatic melanoma, 
which also showed no recurrences in the parotid bed ${ }^{8}$. Reduced parotid bed recurrence is particularly important in the head and neck as optimal control of regional disease and patient quality of life are likely linked and clinically significant.

High-level evidence is lacking to precisely quantify the impact of parotid recurrence on quality of life. However, facial nerve function is important to patient quality of life and House-Brackmann scores have been inversely related to health-related quality of life ${ }^{14}$. It can be inferred that parotid bed recurrence with an associated $42 \%$ risk of facial paralysis in our cohort negatively impacted patient quality of life.

Primary tumor characteristics predictive of parotid bed recurrence after superficial parotidectomy would assist in selecting patients for total parotidectomy. Unfortunately, we were unable to identify any such characteristics in our cohort. Nodal disease burden was greater in patients who recurred in their parotid bed after superficial parotidectomy; however, this information is usually not known until after parotidectomy is complete, so is of limited use in selecting who should undergo total parotidectomy. This is an area where additional research could provide more guidance on selecting patients for total parotidectomy.

It is important to carefully consider the potential for additional morbidity associated with total versus superficial parotidectomy. Given the more extensive facial nerve dissection required for total parotidectomy, postoperative facial nerve function is an important endpoint for consideration. Our results showed no increased facial asymmetry after total parotidectomy compared to superficial parotidectomy. We speculate that total parotidectomy did not result in greater facial nerve injury than superficial parotidectomy because the majority of risk to the facial nerve was during main 
trunk identification and initial dissection to delineate each branch. Deep lobe parotid excision usually occurred after all branches of the facial nerve were already clearly delineated and required only minimal freeing of parotid gland from the underside of the facial nerve. Therefore, there was not significant additional manipulation of the nerve and any additional manipulation occurred with all nerve branches fully visualized. Similarly, other complication rates were equivalent between the two interventions. These findings are consistent with the single previous report of total parotidectomy for melanoma ${ }^{8}$. Increased time required for surgery with subsequent longer anesthetic is another potential source of morbidity. In our cohort, no increased complication rate was identified due to longer surgical time. Still, total parotidectomy does take more time, which may be an important consideration especially in patients with high comorbidities. Finally, increased parotid gland removal results in increased soft tissue deficit. This is important to discuss preoperatively and may impact patient decision making and expectations. Our clinical impression is while a noticeable increase in soft tissue deficit occurs after total parotidectomy, the degree is relatively small and cosmetically acceptable by the overwhelming majority of patients.

Our study has limitations which are important to define for optimal design of future analyses. Given the retrospective nature of this data set, a definitive causal relationship between total parotidectomy and reduced parotid bed recurrence (loss of regional control) cannot be established. It is possible other patient or disease characteristics account for the difference in recurrence rates observed. However, stage and clinically detected versus occult nodal disease were not statistically different between interventions. 
The rate of adjuvant treatment was the same after superficial and total parotidectomy. However, there were differences in the type of adjuvant treatment received. Specifically, interferon was more often given after superficial parotidectomy and radiation after total parotidectomy (Table 1). This may be due to the higher percentage of patients with Stage 3C melanoma in the cohort who underwent total parotidectomy. The authors do not think that the difference in parotid bed recurrence rates is due to this variation in adjuvant treatment because comparison of patients who did and did not recur after superficial parotidectomy showed no difference in adjuvant treatment rate or type.

Median length of follow up was shorter in patients who underwent total parotidectomy (16 months), so it is possible with longer follow up more recurrences would be detected in this group. However, within the superficial parotidectomy group $50 \%$ of the parotid bed recurrences occurred within the first 16 months. Thus, if the interventions had equivalent parotid bed recurrence rates one would expect to see some recurrences after total parotidectomy during the follow-up time of this analysis, of which we had zero. We intend to continue to study our cohort prospectively to allow for further determination of any recurrences and subsequent morbidity.

The retrospective design also affects uniformity of data. Specifically, facial nerve function reporting was variable in presence and quality. However, all patients who underwent total parotidectomy had postoperative facial nerve function documented. While it is possible that patients without documented facial nerve function may have had paresis not included in the analysis, this would serve to increase facial nerve paresis rates after superficial parotidectomy, not total parotidectomy. 
Finally, our data does not address larger questions such as: Does total parotidectomy improve disease free survival or overall survival? Our suspicion is that it does not, but we believe reduction in parotid bed recurrence, and therefore facial nerve paralysis, is compelling enough to consider total parotidectomy over superficial parotidectomy for metastatic melanoma in the parotid gland.

\section{Conclusion}

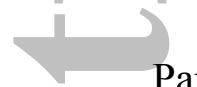

Parotid bed melanoma recurrence was more common after superficial parotidectomy compared to total parotidectomy and recurrence resulted in significant facial nerve functional deficit. Post-operative complications and facial nerve function were comparable after superficial and total parotidectomy. Our results support total parotidectomy when metastatic melanoma involves the parotid nodal basin. Our data also provides a framework for optimal study design in the future to better answer important clinical questions pertaining to surgical treatment of parotid metastatic melanoma.

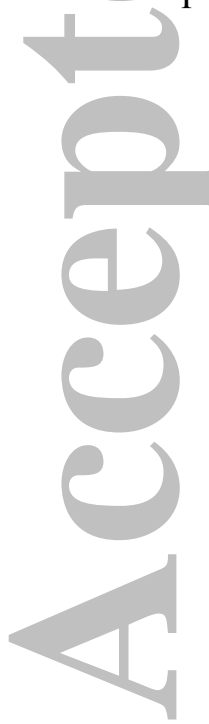

John Wiley \& Sons, Inc. 


\section{References}

1. Siegel RL, Miller KD, Jemal A. Cancer statistics, 2016. CA Cancer J Clin. 2016; $66: 7-30$

2. Miller KD, Siegel RL, Lin CC, et al. Cancer treatment and survivorship statistics, 2016. CA Cancer J Clin. 2016. Epub June 2.

3. Mafee MF: Salivary glands. In: Mafee MF, Valvassori GE, Becker M eds. Valvassori's Imaging of the Head and Neck. 2nd ed. Germany: Thieme; 2012;861.

4. Olsen KD, Moore EJ. Deep lobe parotidectomy: clinical rationale in the management of primary and metastatic cancer. Eur Arch Otorhinolaryngol. 2014; 271:1181-1185.

5. Ergun SS, Gayretli O, Buyukpinarbasili N, et al. Determining the number of intraparotid lymph nodes: postmortem examination. J Craniomaxillofac Surg. $2014 ; 42: 657-660$.

6. Garatea-Crelgo J, Gay-Escoda C, Bermejo B, Buenechea-Imaz R. Morphological study of the parotid lymph nodes. J Craniomaxillofac Surg. 1993; 21:207-209.

7. McKean ME, Lee K, McGregor IA. The distribution of lymph nodes in and around the parotid gland: an anatomical study. Br J Plast Surg. 1985; 38:1-5.

8. Thom JJ, Moore EJ, Price DL, Kasperbauer JR, Starkman SJ, Olsen KD. The role of total parotidectomy for metastatic cutaneous squamous cell carcinoma and malignant melanoma. JAMA Otolaryngol Head Neck Surg. 2014; 140:548-554.

9. Pisani P, Ramponi A, Pia F. The deep parotid lymph nodes: an anatomical and oncological study. J Laryngol Otol. 1996; 110:148-150.

John Wiley \& Sons, Inc.

This article is protected by copyright. All rights reserved. 
10. Bron LP, O'Brien CJ, McNeil EB. Primary and metastatic cancer of the parotid: comparison of clinical behavior in 232 cases. Laryngoscope. 2003; 113:10701075.

11. National Comprehensive Cancer Network. Melanoma (version 3.2015). Available at: https://www.nccn.org/professionals/physician_gls/f_guidelines.asp\#melanoma. Accessed June 14, 2016.

12. Edge S, Carducci M. AJCC Cancer Staging Manual. 7th ed. New York, NY: Springer; 2010.

13. House JW, Brackmann DE. Facial nerve grading system. Otolaryngol Head Neck Surg. 1985; 93:146-147.

14. Kleiss IJ, Hohman MH, Susarla SM, Marres HAM, Hadlock TA. Health-related quality of life in 794 patients with a peripheral facial palsy using the FaCE Scale: a retrospective cohort study. Clin Otolaryngol. 2015; 40:651-656.

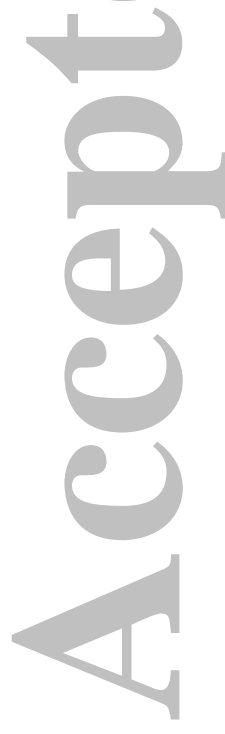

John Wiley \& Sons, Inc.

This article is protected by copyright. All rights reserved. 


\begin{tabular}{|c|c|c|c|}
\hline & $\begin{array}{l}\text { Superficial Parotidectomy } \\
95(74) \text { n (\%) }\end{array}$ & $\begin{array}{l}\text { Total Parotidectomy } \\
34(26) \\
\text { n (\%) }\end{array}$ & $\mathrm{p}$ - value \\
\hline Age at diagnosis & 60 years & 58 years & 0.68 \\
\hline Male & $68(72)$ & $25(74)$ & 1.00 \\
\hline Length of follow up & 24 months & 16 months & 0.05 \\
\hline Stage 3a & $31(33)$ & $8(24)$ & \\
\hline Stage $3 b$ & $35(36)$ & $9(26)$ & \\
\hline Stage 3c & $29(31)$ & $17(50)$ & 0.13 \\
\hline $\begin{array}{l}\text { Clinically detected } \\
\text { disease }\end{array}$ & $40(42)$ & $18(53)$ & 0.32 \\
\hline $\begin{array}{l}\text { Adjuvant treatment } \\
\text { (all) }\end{array}$ & $35(36)$ & $12(35)$ & 1.00 \\
\hline Radiation & $10(11)$ & $8(24)$ & 0.08 \\
\hline Chemotherapy & $0(0)$ & $1(3)$ & 0.26 \\
\hline Immune therapy & $23(24)$ & $2(6)$ & 0.02 \\
\hline Combined & $2(2)$ & $1(3)$ & 1.00 \\
\hline Facial nerve HB 1-3 & $68(97)$ & $30(91)$ & \\
\hline Facial nerve HB 4-6 & $2(3)$ & $3(9)$ & 0.32 \\
\hline Complications & $10(11)$ & $3(9)$ & 1.00 \\
\hline Parotid recurrence & $12(13)[3.4 ; 6.0-19.3]$ & 0 & 0.04 \\
\hline
\end{tabular}

John Wiley \& Sons, Inc.

This article is protected by copyright. All rights reserved. 
Table 1. Comparison of variables by intervention (superficial versus total parotidectomy). Values shown as $\mathrm{N}(\%)$ except for age and length of follow up, which are median values. For parotid recurrence, standard error (SE) with $95 \%$ confidence interval $(\mathrm{CI})$ is shown as [SE; 95\% CI range]. 25 patients who underwent superficial parotidectomy did not have facial nerve function documented. 1 patient who underwent total parotidectomy presented prior to surgery with facial paralysis. These 26 patients were excluded from facial nerve statistical analyses. HB = House-Brackmann.
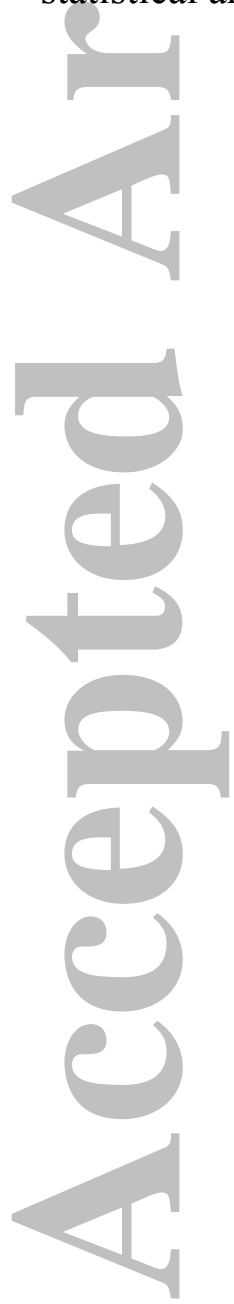

John Wiley \& Sons, Inc. 


\begin{tabular}{|c|c|c|c|}
\hline & $\begin{array}{l}\text { Parotid Recurrence } \\
12(13) n(\%)\end{array}$ & $\begin{array}{l}\text { No parotid recurrence } \\
84(87) \mathrm{n}(\%)\end{array}$ & $\mathrm{p}$ - value \\
\hline \multicolumn{4}{|l|}{ Primary location } \\
\hline Scalp/forehead & $6(50)$ & $27(34)$ & 0.34 \\
\hline Temple/periorbital & $1(8)$ & $16(20)$ & 0.45 \\
\hline Cheek & $4(33)$ & $16(20)$ & 0.45 \\
\hline Ear & $0(0)$ & $17(22)$ & 0.11 \\
\hline Nose/lip & $1(8)$ & $0(0)$ & 0.13 \\
\hline Neck & $0(0)$ & $3(4)$ & 1 \\
\hline \multicolumn{4}{|l|}{ Melanoma subtype } \\
\hline Nodular & $3(30)$ & $22(31)$ & 1 \\
\hline Desmoplastic & $1(10)$ & $4(6)$ & 0.49 \\
\hline \multicolumn{4}{|l|}{ T classification } \\
\hline $\mathrm{T} 1$ & $5(41)$ & $13(17)$ & 0.12 \\
\hline $\mathrm{T} 2$ & $2(17)$ & $18(25)$ & 0.72 \\
\hline $\mathrm{T} 3$ & $3(25)$ & $27(37)$ & 0.53 \\
\hline T4 & $2(17)$ & $15(21)$ & 1 \\
\hline Ulceration present & $2(17)$ & $27(42)$ & 0.12 \\
\hline \multicolumn{4}{|l|}{$\mathrm{N}$ classification } \\
\hline N1 & $4(33)$ & $42(50)$ & 0.36 \\
\hline N2 & $2(17)$ & $25(30)$ & 0.5 \\
\hline N3 & $6(50)$ & $17(20)$ & 0.03 \\
\hline Clinically detected & $4(33)$ & $36(43)$ & 0.76 \\
\hline
\end{tabular}

John Wiley \& Sons, Inc.

This article is protected by copyright. All rights reserved. 


\begin{tabular}{|l|l|l|r|}
\hline disease & & & \\
\hline Satellitosis & $3(50)$ & $7(41)$ & 1 \\
\hline Adjuvant & & & 0.53 \\
\hline Any & & & 1 \\
\hline Radiation & $3(25)$ & $32(38)$ & 0.28 \\
\hline Interferon & $1(8)$ & $9(11)$ & $1(26)$ \\
\hline
\end{tabular}

Table 2. Comparison of variables in patients who did and did not recur after superficial parotidectomy. The primary tumor location was unknown in 5 patients who did not recur in the parotid bed after superficial parotidectomy. A melanoma subtype was not characterized for 14 patients, 12 of which did not recur. The depth of invasion was not documented for 11 primary tumors that did not recur. The presence or absence of ulceration was not documented in an additional 8 patients who did not recur. All percentages and p-values calculated using only cases where this information was provided. Satellitosis is defined by AJCC staging guidelines and thus the denominator for percentages was total N3 disease. Values shown as $\mathrm{N}(\%)$.

John Wiley \& Sons, Inc.

This article is protected by copyright. All rights reserved. 
Figure 1. Parotid bed recurrence-free survival. $p=0.10$
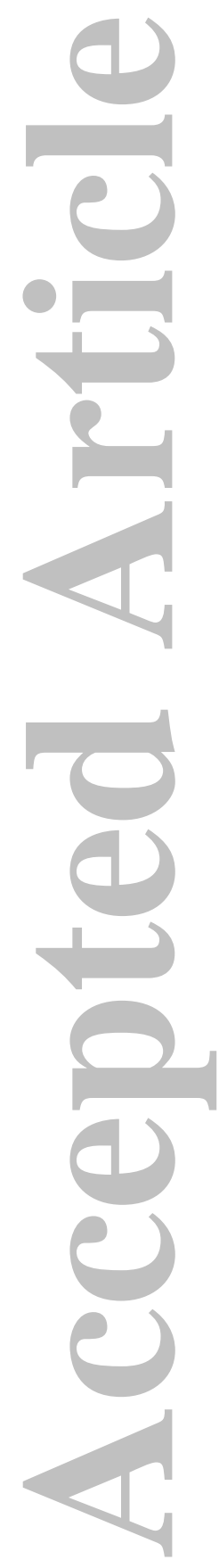

John Wiley \& Sons, Inc.

This article is protected by copyright. All rights reserved. 


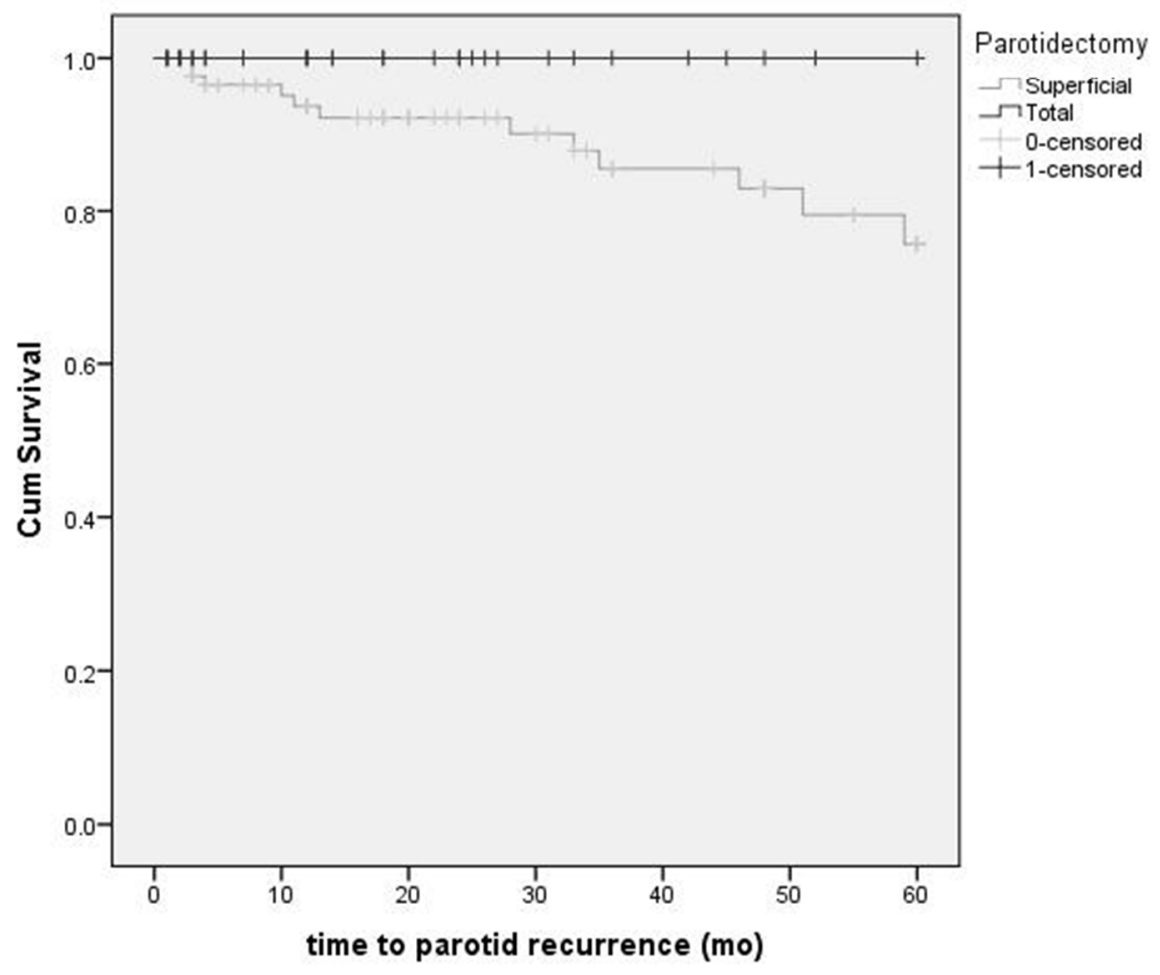

Figure 1. Parotid bed recurrence-free survival. $p=0.10$.

$220 \times 176 \mathrm{~mm}(72 \times 72 \mathrm{DPI})$

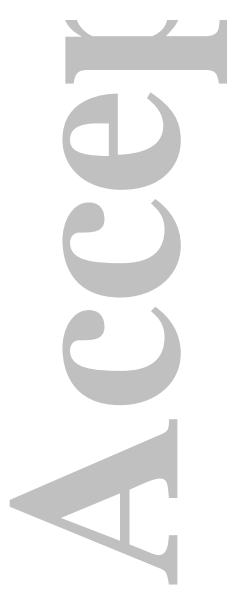

John Wiley \& Sons, Inc.

This article is protected by copyright. All rights reserved. 